\title{
Implementation of a Multi-functional Safety Device for the Automobiles
}

\author{
Dipankar Mitra \\ Chittagong University of \\ Engineering and Technology \\ Dept. of Electrical and \\ Electronic Engineering
}

\author{
Sadaf Yusuf Tahhan \\ Chittagong University of \\ Engineering and Technology \\ Dept. of Electrical and \\ Electronic Engineering
}

\author{
Tajeq Ahmed \\ Chittagong University of \\ Engineering and Technology \\ Dept. of Electrical and \\ Electronic Engineering
}

\begin{abstract}
Safety while plying on personal vehicle as well as in time of goods transportation is a very important aspect. With a view to ensuring these, an attempt is made to build a multifunctional safety device. The term multifunctional over here refers to various form of trouble during the running of vehicle. In this paper a system is developed for automobiles for safety against fire and over load at a time and it is also checked that whether it works properly or not. Above all the cost was also estimated so that it is in the reach of all and be a device better than safety devices already in use.
\end{abstract}

\section{General Terms}

Multifunctional, Safety, Automobiles

\section{Keywords}

PIC 16F877A microcontroller, smoke detection, inclinometer, RF receiver-transmitter, serial communication, ASK modulation and demodulation.

\section{INTRODUCTION}

The system focuses on the safety of automobiles by alerting the drivers as well as the traffic control system about dangers like overload and fire hazard. There are two parts in this system: car side and receiver side. In this system for transmitting the signal radio frequency [6] is used by receivertransmitter module. So, this is a endeavor to enrich transportation facility. In this paper a simple prototype of this useful system is presented.

\section{METHODOLOGY}

The system involves two fundamental sensors, the value of which is sensed by the microcontroller unit (MCU). The program was written to enable two basic hardware modules (ADC module for load sensing and digital I/O for smoke) that read the sensors.

\subsection{Smoke detection}

Here, the idea of smoke detector operation is photoelectric type[10]. A voltage divider[1] is used with a LDR and resistance connected to it. A LED is constantly providing light intensity to the LDR. LM-339 operational amplifier [4] is used as a voltage comparator circuit [2] in this system. This voltage comparator circuit is connected to microcontroller PIC 16F877A [5]. At normal condition, LED is constantly providing light intensity on LDR in an enclosed way. So there is no variation in resistance of LDR and also in the voltage. So according to the reference voltage of operational amplifier the voltage comparator gives output ' 1 ' to microcontroller as a signal of no breakdown. If black, heavy smoke pass between LED and LDR, the resistance of LDR decreases. The voltage comparator will compare the voltage with the reference voltage and the microcontroller will read ' 0 ' as a sign of some kind of breakdown. Here pull-up resistor is used to gain the proper output voltage of the operational amplifier. [4]

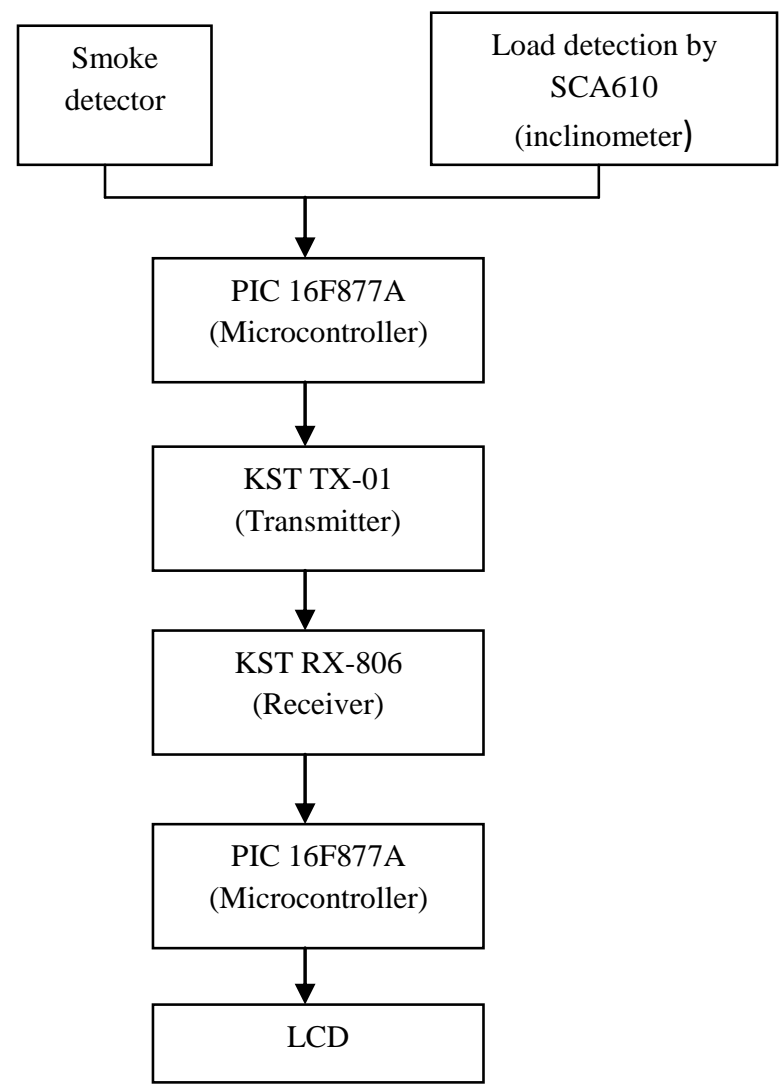

Fig 1: Block diagram of the system

\subsection{Overload Detection}

SCA610 series inclinometer[7] is used as load detector which is connected to the microcontroller through ANO pin of the microcontroller for ADC purpose. This device has a controlled frequency response in the sensing element and $+5 \mathrm{~V}$ supply is reduced for it's operation. No additional components is required because SCA610 has excellent stability over temperature and time, outstanding overload and shock 
durability which makes it suitable for this system. If there is no load variation, the inclinometer does not sense any inclination. It measures the components of gravitational acceleration ' $\mathrm{g}$ '. When there is no overload, it measures the horizontal component of ' $\mathrm{g}$ ' and gives a voltage signal to microcontroller. The microcontroller reads this output as ' 0 ' which is a signal of no breakdown. If load is applied, the inclinometer measures upward or downward components of ' $\mathrm{g}$ ' and it gives a voltage signal to microcontroller. The microcontroller will read the signal as ' 1 ' from inclinometer as a breakdown if it crosses a certain level and detects the vehicle as overloaded [11]. The standard level of load of the vehicles can be changed by changing microcontroller program, as the load is different for different vehicles. The microcontroller will read the variation of voltage. Thus the PIC microcontroller detects the fault and transmits to KST TX01 by serial communication [9].

\subsection{Transmitter Module}

KST TX-01 is a wireless transmitter module which is used because of it's specifications suitable for this process. The operating frequency of $\mathrm{KST} \mathrm{TX}-01$ is $315 \mathrm{MHz}$ to $433.92 \mathrm{MHz}$. It's operating temperature is $-40^{\circ} \mathrm{C}$ to $80^{\circ} \mathrm{C}$, operating voltage is $3 \mathrm{~V}$ to $12 \mathrm{~V}$ and modulation type is amplitude shift keying (ASK). This transmitter module has long distance transmission power of $10 \mathrm{~dB}$ [8]. It is earlier mentioned that the transmission is done by serial communication. Here, Universal Asynchronous ReceiverTransmitter (UART) serial communication is used which transmits data bit by bit. It is used for low volume transmission and the data is transmitted without clock. So it is up to the microcontroller unit to synchronize it's internal baud rate clock to the input data [12].

\subsection{Receiver Module}

Wireless receiver module of KST RX-806 is used in this system. It is a ASK demodulation type circuit. It demodulates the signal transmitted by KST TX-01 and regains the original signal. Strong anti-static protection and high reliability are fantastic features of KST RX-806. It has also receiving sensibility up to $-105 \mathrm{dbm}$ and twice receiving distance compare to others. It has also other very effective characteristics like reasonable bandwidth, ability to suppress co-ordinate frequency, strong anti-jamming and adaptation to all kind of environment. KST RX-806 can suppress assembling and scattering radiation easily and has the ability to restrain the radiation. It has wide temperature range and cam adjust between frequency range for $250 \mathrm{MHz}$ to $450 \mathrm{MHz}$. It increases the ability of anti-disturbing against mobile phone and shape of decoding waves is improved [8].

KST TX-01 transmits its data to KST RX-806 by amplitude shift keying (ASK). ASK is a modulation type which represents digital data as variations in the amplitude of a carrier wave. A finite number of amplitudes is used by ASK and an equal number of bits is each amplitude. The demodulator is designed for the symbol-set used by the modulator. It determines the amplitude of the received signal and sends it back to the symbol it represents. Thus the original data is recovered. In this procedure, frequency and phase of the carrier are kept constant. Both ASK modulation and demodulation processes are relatively cheap. [3][9]

KST RX-806 is connected to another PIC 16F877A microcontroller at receiver side. The microcontroller reads these data from KST RX-806 and a LCD is used for showing the data. There is a scope of using powerful antenna in receiver module to enhance the frequency range of the system.

\section{PROCESSING THE PROGRAMME}

Mikro C for PIC is used for writing the program

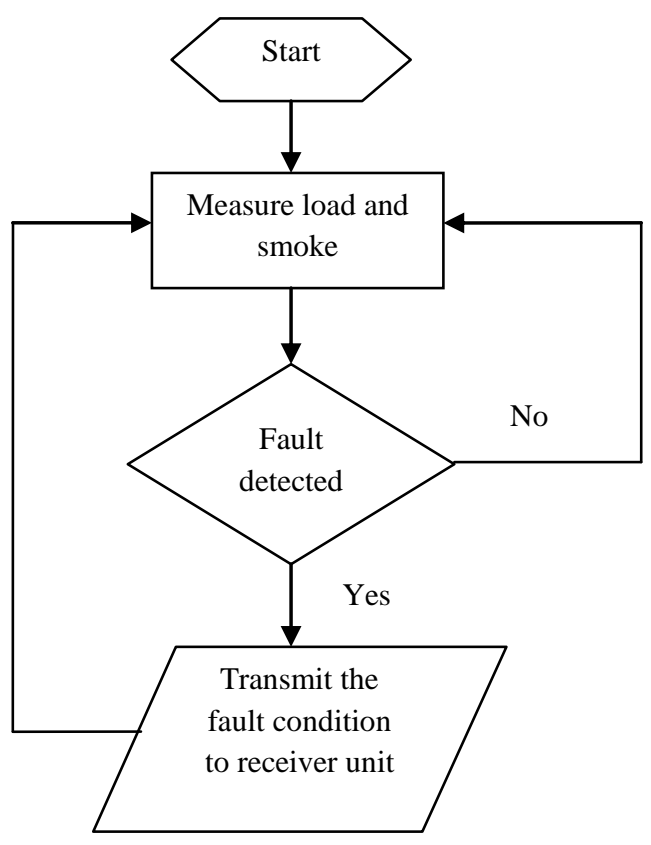

Fig 2: Flow chart of the car side program

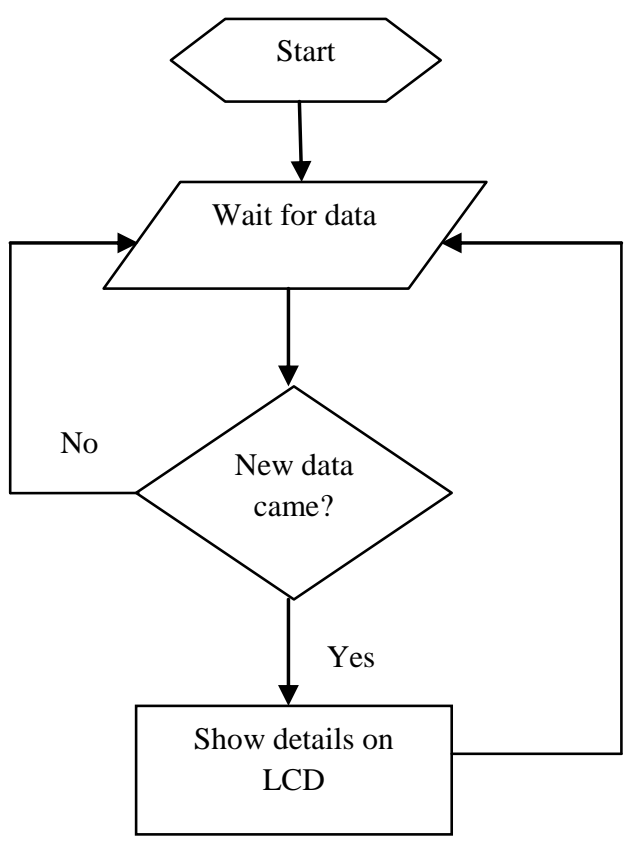

Fig 3: Flow chart of the receiver side program 


\section{IMPLEMENTED CIRCUIT}

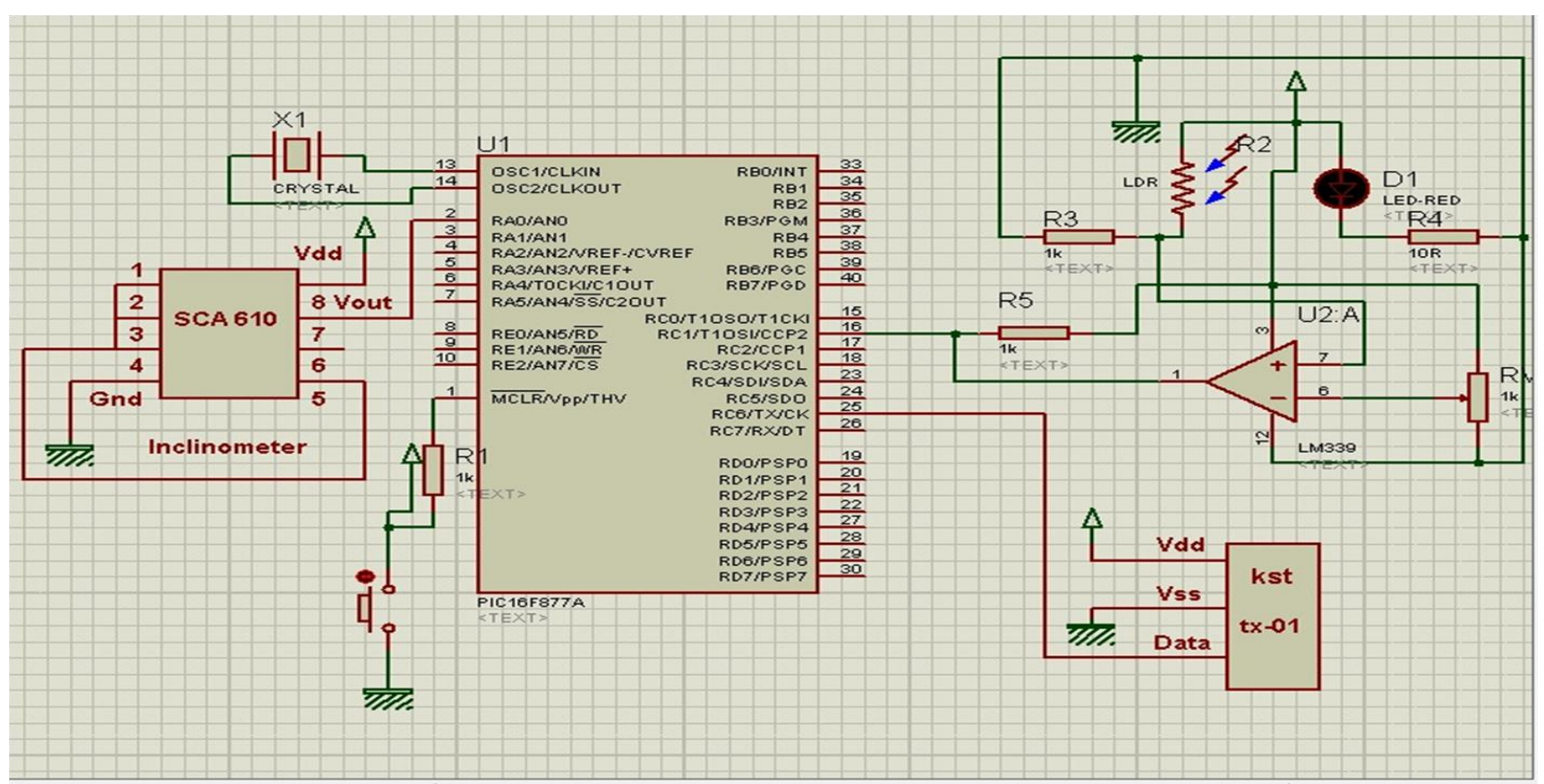

Fig 4: Car side circuit

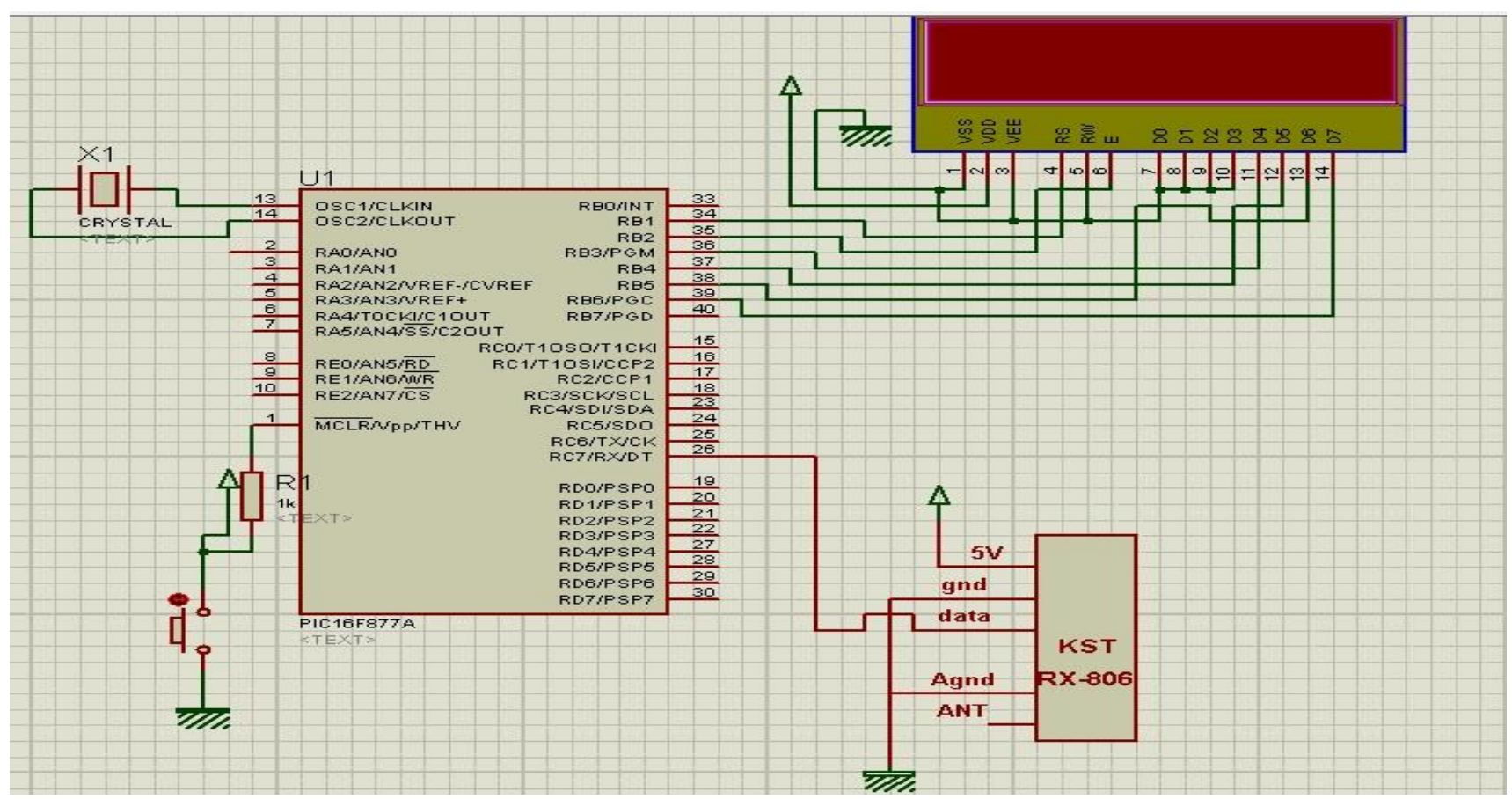

Fig 5: Receiver side circuit

\section{RESULT}

If a vehicle is overloaded the car side part of the system transmits the signal to the receiver side part and LCD shows "Over weight Car no.1" and if any fire hazard occurs in the vehicle again the car side part of the system transmits signal to the receiver part and LCD sows "Smoke detected Car no.1".

\section{CONCLUSION}

This device costing is approximated around USD 65 which is quiet a remarkable in comparison to its uses and application. In this paper protection against fire and overload is ensured and many other safety aspects could also be integrated. In a word there are lots of scopes of development of this device. The signaling system can be improved by GSM module [13] and there is a scope of dedicating the driver's cell phone 
number by which the control room will get much more clear idea about the location of the vehicles.

\section{ACKNOWLEDGEMENTS}

We would like to thank Amit Jyoti Datta, B.S.C in EEE from CUET, Insan Arafat Jamil (ASSRO) and Dr. Quazi Delwar Hossain, Assistant Professor , Dept. of EEE,CUET for their valuable guidance during our thesis work by providing necessary documents.

\section{REFERENCES}

[1] Introductory Circuit Analysis- 11th edition by Robert L. Boylested.

[2] Electronic Device and Circuit Theory- 9th edition by Robert L. Boylested and Louis Nashelsky.

[3] Communication Engineering Fundamentals- 3rd edition by M. Abdus Samad.

[4] Operational Amplifiers and Linear Integrated Circuits- $6^{\text {th }}$ edition by Robert F. Coughlin and Frederick F. Driscoll.
[5] http://www.scribd.com/doc/55435525/PIC16F877A-PinDiagram.

[6] Principles of Electronics-1st edition by V.K.Mehta and Rohit Mehta

[7] SCA610 Datasheet(PDF)-VTI technologies accelerometer/inclinometer.

[8] www.embedded-lab.com

[9] www.wikipedia.com

[10] Applications Guide: System Smoke Detectors-SYSTEM SENSOR

[11] www.riekerinc.com

[12] Serial Communications-SILICON LABS

[13] V.Ramya, B. Palaniappan, "Embedded Technology for vehicle cabin safety Monitoring and Alerting System", International Journal of Computer Science, Engineering and Applications (IJCSEA) Vol.2, No.2, April 2012 